\title{
BMJ Open Factors influencing access to kidney transplantation: a research protocol of a qualitative study on stakeholders' perspectives
}

\author{
Katja Kloss, ${ }^{1}$ Sohal Ismail, ${ }^{1}$ Steef Redeker, ${ }^{1}$ Lothar van Hoogdalem, ${ }^{\odot 1}$ \\ Annemarie Luchtenburg, ${ }^{1}$ Jan J V Busschbach, ${ }^{1}$ Jacqueline van de Wetering ${ }^{2}$
}

To cite: Kloss K, Ismail S, Redeker S, et al. Factors influencing access to kidney transplantation: a research protocol of a qualitative study on stakeholders' perspectives. BMJ Open 2019;9:e032694. doi:10.1136/ bmjopen-2019-032694

- Prepublication history for this paper is available online. To view these files, please visit the journal online (http://dx.doi. org/10.1136/bmjopen-2019032694).

Received 01 July 2019 Revised 12 August 2019 Accepted 13 August 2019

Check for updates

(c) Author(s) (or their employer(s)) 2019. Re-use permitted under CC BY-NC. No commercial re-use. See rights and permissions. Published by BMJ.

${ }^{1}$ Medical Psychology and Psychotherapy, Erasmus Medical Center, Rotterdam, The Netherlands

${ }^{2}$ Nephrology, Erasmus MC University Medical Center, Rotterdam, The Netherlands

Correspondence to

Dr Lothar van Hoogdalem;

I.vanhoogdalem@erasmusmc.nl

\section{ABSTRACT}

Introduction Unequal access to kidney transplantation is suggested, but no systematic inventory exists about factors influencing access to kidney transplantation. There is an absence of any research that has combined stakeholder perspectives along the complete trajectory of transplantation. The present qualitative study explores the contributing factors from the perspectives of multiple stakeholders in this trajectory, including patients, health professionals and health insurance and financial representatives in the Netherlands. Moreover, stakeholders will be invited to suggesting strategies and solutions for handling the facilitating and hindering factors found. By means of interaction, stakeholder groups will arrive at a consensus for new policymaking in the field of a Dutch transplantation care.

Methods and analysis The different stakeholders' perspectives and possible solutions will be explored by interviewing in three phases. In the first phase, stakeholders' group perspectives will be explored with individual interviews and focus group interviews without confrontation of views from other perspectives. In the second phase of focus group interviewing, perspectives will be confronted with the other stakeholders' perspectives assessed. Finally, in the third phase, stakeholders will be invited to focus group discussions for suggesting solutions to overcome barriers and promote facilitators for improving access to transplantation. Approximately, groups from six to twelve participants per focus group and four to maximal six focus groups will be held per stakeholder, depending on the level of saturation, as prescribed by grounded theory. The interviews will be audio-recorded and transcribed verbatim, and qualitative data will be analysed according to the principles of grounded theory supported by using NVivo software. Ethics and dissemination The Medical Ethical Committee of Erasmus MC, Rotterdam, The Netherlands, has approved this study. The results will be disseminated in peer-reviewed journals and major international conferences.

\section{INTRODUCTION}

Unequal access to kidney transplantation is suggested, but no systematic inventory exists about factors influencing access to kidney
Strengths and limitations of this study

- A systematic inventory of factors influencing access to transplantation will be accomplished without a predefined model to allow new information to arise.

- The perspectives of a diversity of stakeholders will be explored including those whose perspectives are rarely represented in literature, ie, policymakers and health insurers.

- Participating in the study will lead stakeholders to generate policy recommendations themselves, facilitating its consequent implementation.

- This study holds the promise of a more equal access to transplantation

The study is limited to the Dutch context.

transplantation. There is an absence of any research that has combined stakeholder perspectives along the complete trajectory of transplantation. ${ }^{1-4}$ The present qualitative study explores the contributing factors from the perspectives of multiple stakeholders in this trajectory, including patients, health professionals and health insurance and financial representatives in the Netherlands. Moreover, stakeholders will be invited to suggesting strategies and solutions for handling the facilitating and hindering factors found. By means of interaction, stakeholder groups will arrive at a consensus for new policymaking in the field of a Dutch kidney transplantation care.

Kidney transplantation is promoted as the best option for renal replacement therapy for a significant group of the patients with end-stage renal disease (ESRD). The advantages of kidney transplantation over dialysis treatment are longer patient survival ${ }^{56}$ and better quality of life. ${ }^{7}$ Further advantages of transplantation with a graft of a living donor compared with a graft of a deceased donor are often better graft quality and graft and patient survival, shorter waiting time, more 
convenient scheduling of the operation and a real chance of pre-emptive transplantation. ${ }^{8-11}$ In the Netherlands, due to the scarcity of organs, pre-emptive transplantation is only possible in practice with an organ from a living donor. Compared with transplanted patients who underwent a longer period of dialysis prior to transplantation, pre-emptive transplantation has additional medical advantages such as longer graft and patient survival, ${ }^{12-15}$ less acute rejection ${ }^{16}$ as well as socioeconomic advantages such as increased rates of patients continuing employment. ${ }^{17}$ Given the advantage of transplantation in general and pre-emptive transplantation in particular, it is remarkable that there are still so many patients on dialysis. Despite the fact that there are patients who are not eligible for transplantation, it could be that a group of patients on dialysis has never been screened for transplantation or missed the opportunity of a pre-emptive transplantation. In this study, we set out to determine whether stakeholders believe that optimal treatment is already established or if there is a perceived inequality.

Equal access to transplantation can be defined in different ways. In this study, we will regard equality in terms of sociodemographic aspects such as migration background and age, medical aspects such as comorbidity or human leukocyte antigens (HLA) and blood type matching and timely aspects such as the work-up process of patients referred to transplantation screening or facilitating the pre-emptive trajectory.

The idea of unequal access to transplantation can be supported by the large prevalence of patients on dialysis waiting for a transplantation from a post-mortem donation. ${ }^{18}$ The problems of organ scarcity and waiting time became partly answered by transplantation with a graft of a living donor. Meanwhile, the percentage of living kidney donations in the Netherlands is among the highest in the world. It has been shown that many patients can find a living donor in their network if more support is given. ${ }^{19}$ Special programme like our National kidney-exchange transplantation programme, $\mathrm{AB} 0$ incompatible and HLA incompatible transplantation and unspecified kidney donation have been established to overcome barriers for (matching) living donation. On one hand, these efforts might have resulted in a saturation of possibilities, and on the other hand still more action can be undertaken to facilitate living donation.

Another indication for inequality in access to transplantation can be that there might be a group of patients who has been on dialysis for years, without even been screened or discussed for transplantation (with the transplantations centres). For the group of patients who has been on dialysis for a short period, a part might have missed the opportunity of pre-emptive transplantation. One of the goals of this investigation is to find the reasons why such non-optimal care persists and how it can be improved.

Observations as the ones above can also be found in the international literature. For instance, there is growing concern that access to transplantation is neither consistent nor equitable. ${ }^{20}$ From the USA and the UK, racial or ethnical ${ }^{21}{ }^{22}$ and age disparities ${ }^{23-25}$ within the patient population are reported as prominent barriers in access to kidney transplantation. Other potential barriers include lower socioeconomic status and poor education leading to a lack of knowledge. ${ }^{26}$

Also in the Dutch renal care setting, patients with a migration background and older patients can be identified as potentially vulnerable groups with respect to access to transplantation. A retrospective analysis of transplant recipients between 2000 and 2011 in our centre revealed that elderly patients and non-European patients were less likely to undergo living donor kidney transplantation. ${ }^{27}$ This would also decrease their chance for pre-emptive transplantation. An analysis of the ethnic composition of our living donor population showed that there were significantly fewer ethnic minority living donors than would be expected based on the (patient) population composition. $^{28}$ The PhD-thesis of M. Laging (2017) showed in a retrospective cohort study that the majority of younger patients $(<25$ years old) were transplanted for 6 years on the regional waiting list. The majority of them was transplanted with a graft from living a donor. In comparison with that only half of the patients in the age group $>65$ years old were transplanted during the same waiting time, from which half of the patients with a graft from a living donor. As a consequence, half of the older population died during the waiting time or had been definitely delisted. The patient group above 65 years old are a relevantly growing group among the patients with ESRD. ${ }^{18}$ Thus, there are several reports about not only unequal access between patients towards optimal care related to logistics and location, but also related to patients' characteristics.

Next to patient-related factors, inequity in access to transplantation may be determined by professional- and system-related factors. For the latter, late referral ${ }^{29} 30$ or the time between diagnosis of the renal disease and transplantation ${ }^{31}$ have been reported to influence access to kidney transplantation. Addressing the disparities in on time access to kidney transplantation including the perspectives of patients, healthcare providers and policymakers has recently been expressed as a research priority in organ transplantation in literature. ${ }^{32}$

Accordingly, the need for policy change and improvement on different system-related factors in the Dutch transplantation care was supported during a recent national meeting organised by the Dutch Transplantation Foundation with medical professionals and donor organisation and patient organisations supported. ${ }^{33}$ Hence, the focus of this study aligns with the need of professionals, donors and patients in The Netherlands. Further, quality indicators for the treatment of chronic kidney failure have been announced by Nefrovisie, the institution for quality management in nephrology in The Netherlands, together with Dutch Kidney Patient Association, Dutch Federation of Nephrology and Zorgverzekeraars Nederland, the umbrella organisation of 10 health insurers in The Netherlands. Pre-emptive kidney transplantation was 
named as one of the quality indicators. The additional question occurs if more patients with ESRD would be eligible for (pre-emptive) kidney transplantation and what the reasons would be for the limited implementation of the quality indicators currently.

There is speculation, but no systematic inventory has yet been accomplished which factors are responsible for the limited implementation of the quality indicators in the treatment of chronic kidney failure and the disparity in access to best practice in renal replacement therapies. It depends, among other aspects, on referral of patients by nephrologists and dialysis centres, preferences of patients and subsequent adequate logistics of the transplantation work-up in transplant centres. This means that access to transplantation depends on multiple stakeholders, multiple motivations and incentives as well as interaction between stakeholders. Given this complex context, a systematic inventory into these factors can best rely on qualitative research methods.

Tong and colleagues ${ }^{34}$ highlighted the value of qualitative research in the field of transplantation, especially when it is about exploring barriers and inequities in access to transplantation. Earlier qualitative research on nephrologists' and patients' perspective on kidney transplantation has been done in Australia and New Zealand. It was demonstrated that physicians aim to optimise outcomes but feel conflicting interests between responsibility for the donor and recipient. ${ }^{35} \mathrm{~A}$ review of nephrologists' perspective on patient referral, eligibility and screening showed that factors such as older age, ethnic background, socioeconomic status, comorbidities and non-adherence influence referral for transplantation. ${ }^{36}$ Patient-related factors that influence the choice of living donor transplantation have been reported to include prioritising own health, guilt and responsibility, ambivalence and uncertainty, seeking decisional validation, needing social support, and cautious donor recruitment. ${ }^{37} 38$ More qualitative research would be helpful to get a better understanding of factors influencing time of referral and perceived inequality in access to transplantation among patients and professionals. Moreover, the perspectives of policymakers and health insurers have yet to be investigated. More insight could help finding strategies to facilitating better timing and a more equal access to optimal treatment.

\section{OBJECTIVES}

The aim of this project is to qualitatively explore factors that influence more optimal access to kidney transplantation from the perspectives of different stakeholders in the Dutch setting, using grounded theory as the qualitative research method. Hence, perspectives of multiple stakeholders will be included, and therefore factors on different levels will be explored, namely psychological, clinical, ethical, social, economic and policy level. The final goal is to identify targets for policy changes and formulating strategies towards improvement of access to kidney transplantation in the Netherlands.

\section{Hypothesis}

Our hypothesis is that different stakeholders have different perspectives on whether there is indeed unequal access, and if such unequal access is assumed, stakeholders hold different views on how the access should be improved. Our hypothesis is further that in reflecting on the perspective of other stakeholders, participants will re-evaluate their point of views in order to reduce disparity. Moreover, in getting closer, they can and will formulate solutions on how to reduce unequal access to transplantation with respect to the views of other stakeholders.

\section{METHODS AND ANALYSIS}

\section{Patient and public involvement}

Patients and most stakeholders were already involved in the study design. As the protocol was a grant proposal of the Dutch Kidney Foundation, the protocol was also judged by the patient reviewers. The study group is regularly advised by an advisory board, which includes representatives of most stakeholders groups involved: nephrologists, policymakers, patients and representatives of the kidney patient association and others. This board helps for instance in thinking about optimal logistical planning for the different groups to keep the research burden for participants as low as possible.

\section{Study population}

The study population will be organised in seven stakeholder groups, namely patients (predialysis, pretransplantation and post-transplantation groups), nephrology and transplantation nurses, social workers, nephrologists, health insurers and policymakers.

Eligible patients are adults ( $>18$ years old) who have 1) been transplanted pre-emptively or non-pre-emptively but underwent dialysis for less than 1 year before transplantation or who have 2) a kidney function of estimated glomerular filtration rate (eGFR) $<15 \mathrm{~mL} / \mathrm{min}$ and for whom initiation of dialysis can be expected within less than 3 months. Additionally, family members and (potential) donors of the kidney patients will be invited to take part in the patient focus groups.

The recruited professional groups will be nephrologists, nephrology and transplantation nurses as well as social workers, representatives of health insurances and policymakers working in the field of kidney transplantation throughout the Netherlands. Policymakers and health insurers will be representatives of national and regional institutions like Dutch Healthcare Authority, Dutch Transplant Foundation, and Dutch Federation of Nephrology and National Healthcare Institute. Within all stakeholder groups, we will strive for sociodemographic variety among the participants to increase the transferability. ${ }^{39}$

\section{Recruitment}

Patients will be recruited from outpatient clinics for nephrology from different treatment centres. The 
eligibility of patients for the study will be assessed by their treating physician before patients are asked to participate in the study. The Dutch patient association and patient gatherings are further sources for approaching patients for participation.

Professionals will be recruited via the professional federations and institutions mentioned above or through different treatment centres, such as the university transplantation centres, the regional hospitals and dialysis centres from different regions. The Dutch Federations of the distinct professional groups will be approached to assist us in reaching out to these professionals. Policy employees or governors from hospitals or governmental institutions, employees, representatives or delegates from health insurance companies will be identified through the Dutch Federations and nephrologists.

During the course of the interviews, we will also consult earlier participants to identify potential participants from the above-mentioned stakeholder groups (snowball sampling).

The senior researchers know a number of the participants from their existing professional network, but a prior acquaintance with the participants was neither an inclusion nor exclusion criterion.

The prospective participants will receive a participant information letter with detailed information about the study and its accomplishment including audio-recording and voluntary participation as well as the informed consent form for signature. Participation is completely voluntary: subjects can leave the study at any time for any reason if they wish to do so without stating any reason and without any consequences for their medical treatment (for patients) or current function (for professionals). If a participant is willing to participate, a study interviewer will invite him or her by telephone or email for the interview.

Our aim is to reduce logistical barriers for all participants as much as possible. Therefore, the focus groups for patients will be held either in the hospital or in a remote location of choice, that is, a community centre. For healthcare professionals, individual interviews will be held at or close to their workplace. Focus group sessions will be held prior to or after regional nephrology or transplantation meetings. Adjunct to this, if necessary, national congress meetings will be used as a venue to conduct the focus groups among healthcare professionals. For policy and health insurance representatives, we will strive to conduct the focus groups at their place of work. Researchers will try to match participants' wishes with the feasibility of the study. In coordination with the participant, an appointment and location for the interview will be assigned. It could be that different settings (location, number and type of co-participants, time of the year, etc.) might provoke different responses. We aim at minimising such variations and maximising the input that participants can and are willing to give. Therefore, the interviewers are trained and consequently (monthly) supervised to stimulate the responses/discussions in such a way that maximal saturation can be reached (ie, making sure that the participant can maximally elaborate on all presented/emerging topics).

\section{DESIGN AND PROCEDURE}

Participants will be invited to take part in an individual interview or a focus group interview. The choice of using both group and individual interviews makes it possible to profit from the advantages of both methods: focus group interviews facilitate a broader exploration, whereas individual interviews allow a more in-depth exploration of topics raised. ${ }^{40}$ Moreover, an advantage of focus group interviews is that they allow insight into complex behaviour and motivation due to the interaction happening during the focus groups. ${ }^{41}$ Additionally, applying both individual and focus group interviews will allow to test themes emerged from individual interviews in a broader range of respondents in the focus groups in short time. ${ }^{40}$ This will increase credibility of the study results. ${ }^{39}$

\section{Three phases}

The interviews will be conducted in three phases. In the first phase, we will try to gather as much information as possible about factors that influence the access to transplantation according to the stakeholder groups by proceeding individual or focus group interviews. Then, focus groups will be organised separately for pre-dialysis and pretransplantation or post-transplantation patients, nephrologists, nurses, social workers, policymakers and insurance representatives. This homogeneity within the focus groups will ensure that participants are able to raise issues for discussion. Too much heterogeneity will inhibit discussion, especially when there are status distinctions between participants. ${ }^{42}$

In the second phase, focus groups will be held with the same stakeholder groups as in the first phase. In the beginning, stakeholders will be confronted with the outcomes of the first phase from their own but also from the other stakeholder groups. The objective of this phase is to let participants of each group reflect on the factors mentioned by their group but also by stakeholders from the other groups, on what factors they think influence accesses to transplantation. The expectation is that due to this iterative process, a more integrated model can be generated of the factors that can influence access to transplantation, from different perspectives.

In the third phase, focus group sessions will be held with the same respective stakeholder groups from the previous two phases. Again, results from the previous phase will be presented in the beginning. The main objective of these focus groups will be to generate solutions for the integrated hindering or facilitating factors that emerged in the second phase. Participants who already took part in one phase will be invited to return to another phase of the study. New participants will also be welcomed to further prevent dropout. 
The three phases of this study will each encompass approximately 1 year. The first 1 or 2 months of each year will be used to write down a detailed script for conducting the focus groups. The last 1 or 2 months of each year will be used for transcribing and analysing the data. The remaining 8 to 10 months will be used for the most important part of this study, namely recruiting participants for the interviews and conducting the individual interviews or focus groups, respectively. The fourth year will mainly be used for reporting and disseminating the outcomes.

\section{Data collection}

\section{Qualitative interviews}

The individual interviews and the focus group interviews will be conducted by a moderator. These will be the junior researcher in Medical Psychology, a transplantation coordinator, or a senior researcher in Medical Psychology, with assistance of one of the other colleagues who will take back-up paper and pencil notes and will monitor the discussed topics. All researchers have knowledge of qualitative methods based on previous research projects and training. The interviews will be audio-recorded after signed informed consent of the participant. If a participant does not master the Dutch language, the use of a translated questionnaire and an interpreter for interviews will be considered.

The interviews will last about $45 \mathrm{~min}$ to $1.5 \mathrm{~h}$ for the individual interviews and about 2 to $3 \mathrm{~h}$ for focus groups, respectively. Participants will be asked what their attitudes, opinions, preferences and beliefs are regarding access to transplantation. The interviews will cover the topics clinical, psychological, social, ethical, economic and policy factors influencing access to transplantation. The moderators will make use of an interview guide to monitor the topics discussed and to be able to offer prompts or sub-questions to a topic in case a participant does not raise a topic by himself or cannot come up spontaneously with any thoughts on one topic.

During the focus group sessions, the moderator will also use a flipchart to make notes for the group. The mediator will keep the discussion going and make sure that every participant gets the chance to contribute. At the end of a focus group session, the mediator will hold a debriefing, that is a summary of the raised issues. Finally, the participants will be asked to fill in a short questionnaire about their sociodemographic characteristics, their type of therapy (for patients) or their current function (for professionals). If necessary, the questionnaire is offered in other languages than Dutch.

Both types of interviews will be transcribed verbatim and the names of the participants will be replaced with identification codes to assure anonymity. If necessary, transcriptions will be translated into the Dutch language. We will not return the transcripts to participants for comments or corrections. There is no evidence that the use of certain checks improves research quality where the primary purpose of the research is theory development. ${ }^{43}$

\section{Data analysis}

Data from the interviews will be analysed according to the principles of grounded theory. ${ }^{44}$ Using this inductive approach, a model or theory will be developed on the basis of the qualitative data generated. This approach is chosen as some perspectives of stakeholder groups have never been studied and because an integrated model based on the perspectives of the multiple stakeholders has never been formulated.

Two researchers will read through the interview transcripts independently and data-driven codes will be assigned based on words and phrases. ${ }^{45}$ This process goes on until the two researchers separately worked through the whole transcript. The steps of open, axial and selective coding will be taken. Therefore, codes will be combined in order to generate a covering category. The two researchers then jointly cluster the derived categories into themes. Thereby, they identify the underlying uniformities of the categories and further sharpen the conceptual structure of each theme. During analysis, it is also important to measure the extensiveness (frequency, intensity and specificity) in which a topic is discussed. Consequently, a grid will be generated according to Miles and Huberman's method in order to be able to compare the data between the groups. ${ }^{46}$ Within each theme, responses are evaluated across the different study groups to search for similarities and differences. Finally, the themes derived will be discussed in the research team. The qualitative software programme NVivo (v12) will be used during analysis to maximise efficiency.

Data generated by interviewing will be analysed until no new concepts or information arises, that is, data saturation is reached following a general principle in qualitative research. ${ }^{47}$ In other words, data saturation is reached when there is enough information to replicate the study, ${ }^{48}$ and when further coding is no longer feasible. ${ }^{45} 49$ Hence, we will continue inclusion and data analysis until data saturation has been reached per stakeholder group. To monitor this continuously, each interview will be transcribed as soon as possible and initial coding carried out before the subsequent interview. Data collection will cease for that specific stakeholder group if no new themes have emerged. $^{49}$

Reporting of qualitative findings will adhere to the consolidated criteria for reporting qualitative research (COREQ). ${ }^{50}$

\section{Sample size calculation}

We did an effort to calculate the sample size on the basis of focus groups only. The focus groups will be organised homogeneous, thus, subdivided in the seven study groups. Four to six focus groups will be held per study group ${ }^{40}$ or until data saturation has been achieved ${ }^{45}{ }^{49}$ We will strive for a maximum of 12 participants per focus group, with a minimum of six participants. ${ }^{42}$ Hence, if four to six focus groups per stakeholder group are needed with seven study groups of stakeholders in each phase, we have to organise $(4 \times 7=) 28$ to $(6 \times 7=) 42$ focus group sessions per 
phase. If six to twelve participants will participate in each focus group, $(6 \times 28=)$ minimal 168 to $(12 \times 42=)$ maximal 504 participants will take part in this study. We aim to recruit 24 to 72 participants per study group. Considering the drop-out rate in the focus groups, approximately 135 participants must be approached per study group ${ }^{51}$ If saturation is reached earlier, less participants need to be recruited. Moreover, diversity among the stakeholder groups has to be considered. For instance, it is not yet known if 135 health insurers or health policymakers are indeed involved in kidney transplantation in a way relevant for this investigation and if they are offering individually such diverse perspectives, that recruitment has to be continued to the maximum of the expected participants.

\section{Status of the trial}

The study started in May 2018 and participant recruitment and initial data analyses are ongoing. The trial will last until December 2023.

\section{ETHICS AND DISSEMINATION}

The Medical Ethical Committee of Erasmus MC, Rotterdam, The Netherlands, has approved this study, registered under MEC-2018-1473. Given the non-interventional nature of this study, a study registration was considered but deemed unnecessary by the Medical Research Involving Human Subjects Act. Participants will be given full study information (with a consideration period of minimum 1 week) and asked to provide written informed consent if they wish to participate.

The study will be conducted in accordance with the Declaration of Helsinki (Tokyo, Venice, Hong Kong and Somerset West amendments). A sequential identification number will be automatically attributed to each patient that has given consent to participate in the study. This number will identify the patient and must be included on all documents. Only the main researcher can link the code to the patient's identity.

Acknowledgements We wish to thank Dutch Kidney Foundation for funding this study.

Contributors JJVB, SYI, JvdW and SR made substantial contributions to conception and design. KK, SYI, JJVB, AL, LEvH and JvdW have been involved in drafting the manuscript or revising it critically for important intellectual content. All authors read and approved the final manuscript.

Funding This research is funded by Dutch Kidney Foundation.

Competing interests None declared.

Patient consent for publication Not required.

Provenance and peer review Not commissioned; externally peer reviewed.

Open access This is an open access article distributed in accordance with the Creative Commons Attribution Non Commercial (CC BY-NC 4.0) license, which permits others to distribute, remix, adapt, build upon this work non-commercially, and license their derivative works on different terms, provided the original work is properly cited, appropriate credit is given, any changes made indicated, and the use is non-commercial. See: http://creativecommons.org/licenses/by-nc/4.0/.

\section{REFERENCES}

1. Dudley CRK, Johnson RJ, Thomas HL, et al. Factors that influence access to the National renal transplant waiting list. Transplantation 2009;88:96-102.

2. Vamos EP, Novak M, Mucsi I. Non-Medical factors influencing access to renal transplantation. Int Urol Nephrol 2009;41:607-16.

3. Oniscu GC, Schalkwijk AA, Johnson RJ. Equity of access to renal transplant waiting list and renal transplantation in Scotland: cohort study. BMJ 2003;327.

4. Ismail SY, Luchtenburg AE, Kal-V Gestel JA, et al. Modifiable factors in access to living-donor kidney transplantation among diverse populations. Transplantation 2013;96:586-90.

5. Edwards EB, Bennett LE, Cecka JM. Effect of HLA matching on the relative risk of mortality for kidney recipients: a comparison of the mortality risk after transplant to the mortality risk of remaining on the waiting list. Transplantation 1997;64:1274-7.

6. Wolfe RA, Ashby VB, Milford EL, et al. Comparison of mortality in all patients on dialysis, patients on dialysis awaiting transplantation, and recipients of a first cadaveric transplant. N Engl J Med 1999;341:1725-30.

7. Ogutmen B, Yildirim A, Sever MS, et al. Health-Related quality of life after kidney transplantation in comparison intermittent hemodialysis, peritoneal dialysis, and normal controls. Transplant Proc 2006;38:419-21.

8. Janki S, Klop KWJ, Kimenai HJAN, et al. Long-Term follow-up after liVE kidney donation (love) study: a longitudinal comparison study protocol. BMC Nephrol 2016;17:14.

9. Liem YS, Weimar W. Early living-donor kidney transplantation: a review of the associated survival benefit. Transplantation 2009;87:317-8.

10. Joo KW, Shin SJ, Lee SH, et al. Preemptive transplantation and longterm outcome in living donor kidney transplantation, single-center experience. Transplant Proc 2007;39:3061-4.

11. Maggiore U, Budde $\mathrm{K}$, Heemann U, et al. Long-Term risks of kidney living donation: review and position paper by the ERA-EDTA Descartes Working group. Nephrology Dialysis Transplantation 2017;32:216-23.

12. Asderakis A, Augustine T, Dyer P. Pre-Emptive kidney transplantation: the attractive alternative. Nephrol Dial Transplant 1998;13:1799-803.

13. Yoo SW, Kwon OJ, Kang CM. Preemptive living-donor renal transplantation: outcome and clinical advantages. Transplant Proc 2009;41:117-20.

14. Meier-Kriesche $\mathrm{H}-\mathrm{U}$, Port FK, Ojo AO, et al. Effect of waiting time on renal transplant outcome. Kidney Int 2000;58:1311-7.

15. Malho A, Malheiro J, Fonseca I, et al. Advantages of kidney transplant precocity in graft long-term survival. In: Transplantation proceedings: 2012. . Elsevier, 2012: 44. 2344-7.

16. Mange KC, Joffe MM. Dialysis prior to living donor kidney transplantation and rates of acute rejection. Nephrology Dialysis Tranplantation 2003;18:172-7.

17. Abou Ayache R, Bridoux F, Pessione F, et al. Preemptive renal transplantation in adults. In: Transplantation proceedings: 2005. Elsevier, 2005: 37. 2817-8.

18. Nefrovisie. Nierfunctievervangende behandeling in Nederland. Jaarboek 2017, 2018.

19. Ismail SY, Luchtenburg AE, Timman R, et al. Home-Based family intervention increases knowledge, communication and living donation rates: a randomized controlled trial. Am J Transplant 2014;14:1862-9.

20. Batabyal P, Chapman JR, Wong G, et al. Tong A: clinical practice guidelines on Wait-Listing for kidney transplantation: consistent and equitable? Transplantation 2012;94:703-13.

21. Purnell TS, Luo X, Cooper LA, et al. Association of race and ethnicity with live donor kidney transplantation in the United States from 1995 to 2014. JAMA 2018;319:49-61.

22. Hall EC, Massie AB, James NT, et al. Effect of eliminating priority points for HLA-B matching on racial disparities in kidney transplant rates. Am J Kidney Dis 2011;58:813-6.

23. Wu DA, Robb ML, Watson CJE, et al. Barriers to living donor kidney transplantation in the United Kingdom: a national observational study. Nephrol Dial Transplant 2017;32:890-900.

24. Purnell TS, Hall YN. Understanding and overcoming barriers to living kidney donation among United States racial and ethnic minorities. Advances in Chronic Kidney Disease 2012;19:244-51.

25. Gore JL, Danovitch GM, Litwin MS, et al. Disparities in the utilization of live donor renal transplantation. Am J Transplant 2009;9:1124-33.

26. Coorey GM, Paykin C, Singleton-Driscoll LC, et al. Barriers to preemptive kidney transplantation. Am J Nurs 2009;109:28-37. quiz 38. 
27. Roodnat JI, Laging M, Massey EK, et al. Accumulation of unfavorable clinical and socioeconomic factors precludes living donor kidney transplantation. Transplantation 2012;93:518-23.

28. Roodnat JI, van de Wetering J, Zuidema W, et al. Ethnically diverse populations and their participation in living kidney donation programs. Transplantation 2010;89:1263-9.

29. Cass A, Cunningham J, Snelling P, et al. Late referral to a nephrologist reduces access to renal transplantation. Am J Kidney Dis 2003;42:1043-9.

30. Jungers $P$, Joly $D$, Nguyen-Khoa $T$, et al. Continued late referral of patients with chronic kidney disease. Causes, consequences, and approaches to improvement] Retard persistant au suivi nephrologique de l'insuffisance renale chronique. Causes, consequences et moyens d'amelioration. Presse Med 2006;35:17-22.

31. Helmick RA, Jay CL, Price BA, et al. Identifying barriers to preemptive kidney transplantation in a living donor transplant cohort. Transplant Direct 2018;4:e356.

32. Tong A, Sautenet B, Chapman JR, et al. Research priority setting in organ transplantation: a systematic review. Transplant International 2017;30:327-43.

33. Dutch Transplant Foundation. Leiden: Nederlandse Transplantatie Stichting, 2017. Available: http://www.transplantatiestichting.nl [Accessed 31 May 2017].

34. Tong A, Chapman JR, Israni A, et al. Qualitative research in organ transplantation: recent contributions to clinical care and policy. Am J Transplant 2013;13:1390-9.

35. Hanson CS, Chadban SJ, Chapman JR, et al. Nephrologists' perspectives on recipient eligibility and access to living kidney donor transplantation. Transplantation 2016;100:943-53.

36. Tong A, Hanson CS, Chapman JR, et al. The Preferences and Perspectives of Nephrologists on Patients' Access to Kidney Transplantation. Transplantation 2014;98:682-91.

37. Hanson CS, Chadban SJ, Chapman JR, et al. The expectations and attitudes of patients with chronic kidney disease toward living kidney donor transplantation: a thematic synthesis of qualitative studies. Transplantation 2015;99:540-54.

38. van Hoogdalem LE, Hoitsma A, Timman R, et al. Shared DecisionMaking in Kidney Patients: Involvement in Decisions Regarding the
Quality of Deceased Donor Kidneys. In: Transplantation proceedings: 2018. . Elsevier, 2018: 50. 3152-9.

39. Schwandt TA, Lincoln YS, Guba EG. Judging interpretations: but is it rigorous? trustworthiness and authenticity in naturalistic evaluation. New Directions for Evaluation 2007;2007:11-25.

40. Morgan DL. Focus groups. Annu Rev Sociol 1996;22:129-52.

41. Morgan DL, Krueger RA. When to use focus groups and why. Successful focus groups: Advancing the state of the art 1993;1:3-19.

42. Krueger RA. Focus groups: a practical guide for applied research. Sage publications, 2014

43. Thomas DR. Feedback from research participants: are member checks useful in qualitative research? Qual Res Psychol 2017;14:23-41

44. Strauss A, Corbin J. Grounded theory methodology. Handbook of qualitative research 1994;17:273-85.

45. Corbin J, Strauss A. Strategies for qualitative data analysis. basics of qualitative research techniques and procedures for developing grounded theory, 2008.

46. Miles MB, HUBERMAN AM. Drawing valid meaning from qualitative data: toward a shared craft. Educational Researcher 1984;13:20-30.

47. Glaser BG. Strauss al: the constant comparative method of qualitative analysis. The discovery of grounded theory: Strategies for qualitative research 1967;101.

48. O’Reilly M, Parker N. 'Unsatisfactory Saturation': a critical exploration of the notion of saturated sample sizes in qualitative research. Qualitative Research 2013;13:190-7.

49. Guest $\mathrm{G}$, Bunce A. Johnson L: how many interviews are enough? an experiment with data saturation and variability. Field methods 2006;18:59-82.

50. Tong A, Sainsbury P, Craig J. Consolidated criteria for reporting qualitative research (COREQ): a 32-item checklist for interviews and focus groups. International Journal for Quality in Health Care 2007;19:349-57.

51. Tausch AP. Methodological aspects of focus groups in health research: results of qualitative interviews with focus group moderators. Glob Qual Nurs Res 2016;3. 